its undamaged areas can foster normal levels of other cognitive abilities.

Uncorrelated cognitive abilities are significant despite not being bundled up in $g$. People today are better at lateral thinking on the job and better at chess, and more acute in on-the-spot assessment of the quality of political debate, than they used to be (Flynn, in press). Brain-damaged children can keep up at school despite below-average Fpsa. If the theory of intelligence is to accommodate uncorrelated abilities, it must transcend $g$. What form would such a theory of intelligence take?

It would have three levels: (1) The B (brain) level, where brain physiology shows how much coordination and how much autonomy functional mental abilities are likely to manifest - shows what degrees and kinds of problem-solving abilities are likely for both normal and abnormal brains. (2) The ID (individual differences) level, where we assess how cognitive abilities vary from one person to another in a homogeneous social setting, and which shows the extent to which abilities are inter-correlated and predictive of one another in that context. (3) The S (social) level, where evolving and diverse social priorities over time free specific mental abilities from the strictures of $g$ (within the limits that the brain allows) and shows them swimming freely from one another and having important consequences. It shows, for example, why America, despite a huge increase in Fpsa, has to import foreigners to do its mathematics.

An affection for acronyms suggests a label like the BIDS theory of intelligence. Its focus would be making sense of how various levels are interrelated. At times, one level may show that what happens on another is surprising enough to require explanation. For example, brain physiology (B level) suggests that Fpsa is functionally independent of other mental abilities. Yet, when we measure individual differences (ID level), $g$ emerges - which is to say individuals who beat the rest of us on one cognitive skill, often outdo us on most cognitive skills. So we have to go back to the brain. Even though different areas are autonomous in the sense that one can function when another is damaged, and in the sense that they can be differentially developed by social change, there must be some overall qualitative factor (the synapses?) - something that makes one normal brain function better on virtually all kinds of problemsolving than another.

At other times, one level may even imply that what is happening on another level is impossible. For example, Jensen (1998, pp. 445-58) analyzed twin studies on the ID (individual differences) level that dramatized the weakness of environment. Indeed, environment appeared so weak that environmental change could not possibly cause huge cognitive gains over a short time - which seemed to imply that IQ gains simply had to have a genetic origin (hybrid vigor) or be spurious. At this point, the Dickens-Flynn model restored coherence to the system by showing that the primacy of genes over environment in individual life history is fully compatible with huge environmental effects as society evolves (Dickens \& Flynn 2001a; 2001b).

The $g$ theory of intelligence is limited because it views the physiological and sociological levels through its own spectacles. It asks: What elementary cognitive tasks correlate with $g$; what cognitive trends over time correlate with $g$ or at least are factor invariant (Wicherts et al. 2004); and so forth. It is as though the physics of moving objects within the earth's gravitational sphere had demanded that astronomy and subatomic physics confine themselves to its model, whereas the way forward was more comprehensive models within which Galileo's equations found their proper place.

The BIDS theory has already paid dividends. Schools teach young children matrices under the delusion that matrice skills and arithmetic reasoning are functionally related. IQ gains over time on the Wechsler Intelligence Scale for Children (WISC) subtests show that the first skill can be greatly augmented with no effect on the second. The relationship is actually correlational. It is as though we observed that good high jumpers tend to be better-than-average sprinters at any given time - and drew the conclusion that the way to improve high-jump performance was to practice sprinting. We would quickly discover that no high jumper hurtles toward the bar at maximum speed; rather, one gathers the moderate amount of momentum compatible with timing the jump. The correlation between high-jump excellence and sprinting excellence does not signal a functional relationship between the two skills.

A symposium should be convened so that Blair and likeminded thinkers and $g$-men (because $g$ is still important on its proper level) can get together and look for a breakthrough in the theory of intelligence. We live in exciting times.

\section{Early intervention and the growth of children's fluid intelligence: A cognitive developmental perspective}

\section{Ruth M. Ford}

Department of Psychology, University of Wales Swansea, Swansea SA2 8PP, United Kingdom

r.ford@swansea.ac.uk

Abstract: From the stance of cognitive developmental theories, claims that general $g$ is an entity of the mind are compatible with notions about domain-general development and age-invariant individual differences. Whether executive function is equated with general $g$ or fluid $g$, research into the mechanisms by which development occurs is essential to elucidate the kinds of environmental inputs that engender effective intervention.

The debate surrounding the existence of general $g$, and its relation to fluid $g$, bears on the efforts of cognitive developmental psychologists to distinguish between general and specific aspects of children's intellectual growth (Case et al. 2001; Lautrey 2002). Domain-general approaches to development aim to identify cognitive skills that exert a pervasive influence on behavior, even in the presence of specialized abilities with which they interact. In contrast, domain-specific approaches offer a compartmentalized view of the mind by focusing exclusively on the operation of functionally independent modules.

A variety of domain-general accounts exist, some of which have advocated components of executive function, such as working memory, as prime candidates for explaining broad, agedependent gains in intellectual ability (e.g., Case 1992). Despite their physiological localization in the frontal regions of the brain, executive functions could thus constitute a driving force in cognitive development that has ramifications for all mental activities. Recent years have seen major advances in the understanding of executive function and its role in the emergence of consciousness (Zelazo 2004), agency (Russell 1999), and self-regulation (Carlson 2003). Not only does executive function undergo marked improvements as children grow older, the distinction between "hot" and "cool" executive function seems well placed to provide new insights into the development of social cognition and behavior (Zelazo et al. 2005).

If executive function is equated instead with fluid $g$, then general $g$ might correspond with some other aspect of development such as global processing speed (e.g., Kail 1991) or, alternatively, a dimension of intelligence that is not related to development at all. As an example of the latter approach, the minimal cognitive architecture model of intelligence and development (Anderson 2001) views intelligent behavior as a product of both age-invariant and developmental mechanisms. The model assumes that age-invariant mechanisms are responsible for individual differences in intellectual ability within a particular developmental level and are determined mainly by heredity. In contrast, it sees developmental mechanisms as involving the maturation of dedicated information-processing 
modules, including executive function, that are more amenable to environmental influences.

Uniformly, theories of cognitive development posit that information provided by the environment is necessary for cognitive growth and, thus, that experiential factors have an impact on developmental outcomes. The assumption is that, whereas heredity might place limits on a child's potential achievements, it is the environment that determines the extent to which this potential is realized (Sternberg 2002). Relative to the extensive study of the heritability of general $g$, there has been little investigation of whether specific cognitive functions are differentially sensitive to environmental stimulation (Grigorenko \& Sternberg 2003). To distinguish between varieties of domain-specific development, however, is to acknowledge the possibility that some cognitive functions exhibit greater plasticity than others (Flavell et al. 2002).

Language and executive skills in young children are affected more severely by low socio-economic status than are other measures of intellectual ability (Noble et al. 2005), suggesting they represent vulnerable aspects of cognitive development that might profitably be targeted by intervention. Importantly, as reviewed in the target article, what limited evidence is available indicates that the development of executive function during early childhood is probably responsive to nurture. Such evidence bolsters the recommendation that early compensatory education programs for disadvantaged children should strive not only to impart knowledge, but to foster those thinking skills that enable children to use their knowledge effectively.

With the aim of intervention in mind, an important agenda for future research is to examine the basic mechanisms driving cognitive development (i.e., an emphasis on the how rather than what of development; Case \& Mueller 2001; Siegler 1996) to improve the efficacy of such programs. Potentially, the goal of improving executive function in children with low socio-economic status could be accomplished by encouraging their parents to interact with them in ways that promote language acquisition and self-regulation. Gauvain (2001) argued that social processes qualify as mechanisms of intellectual growth on the grounds that they teach children strategies for acquiring and manipulating knowledge, for choosing between alternative courses of action, and for deploying their knowledge and skills in new contexts. From a Vygotskian perspective, children learn to think and to regulate their behavior by internalizing the language of more competent members of their community during social interactions that involve problem solving. The theory thus assumes that children experience new ways of thinking first collaboratively and then on the individual plane (Vygotsky 1978). The view that social processes are integral to cognitive development is compatible with Siegler's (1996) concepts of variability and choice as mechanisms of learning, as well as the hypothesized role of private speech in aspects of executive function (Carlson 2003).

Those of us involved with early intervention know that it is costly and time consuming, and often regarded less favorably by funding bodies than is research deemed "pure" rather than "applied." In concluding, I therefore want to emphasize that the use of longitudinal methods in early intervention presents a prime opportunity for addressing fundamental questions about the nature of children's cognitive development. Longitudinal research has the potential to shed light on causal mechanisms in cognitive development, for example, those underpinning the close relations between executive skills, language, and theory of mind (Carlson et al. 2004). By incorporating precise manipulations of children's intervention experiences into longitudinal assessments of their cognitive and social/emotional functioning it should be possible to elucidate both the development of different aspects of intelligence during early childhood and the relations between emotion and higher-order cognition that traditionally have been neglected by cognitive developmental researchers.

\section{There is more to fluid intelligence than working memory capacity and executive function}

\author{
Dennis Garlick and Terrence J. Sejnowski \\ Computational Neurobiology Laboratory, Salk Institute, La Jolla, CA 92037. \\ dgarlick@salk.edu terry@salk.edu http://www.cnl.salk.edu
}

Abstract: Although working memory capacity and executive function contribute to human intelligence, we question whether there is an equivalence between them and fluid intelligence. We contend that any satisfactory neurobiological explanation of fluid intelligence needs to include abstraction as an important computational component of brain processing.

Understanding fluid intelligence is a fascinating problem for behavioral and brain research. Fluid intelligence problems such as Raven's Progressive Matrices, Number Series, and Word Analogies involve presenting participants with problems that they are unlikely to have seen before. Successful performance cannot then be attributed to any simple learning mechanism based on previously seeing and memorizing the correct answer to the exact same problem. However, despite this, humans are able to solve these kinds of problems, suggesting that fluid intelligence is an important construct for assessing the human capacity to perform successfully across a wide range of situations. This is also supported by psychometric findings suggesting that fluid intelligence is the best predictor of performance in situations that involve human intelligence, including performance at school, at university, and in cognitively demanding occupations (Gottfredson 1997).

Understanding the nature of fluid intelligence has been a profound problem for psychometric intelligence research. Indeed, even recent reviews admit that we still have no satisfactory explanation of what causes differences in fluid intelligence (Brody 1992; Jensen 1998; Neisser et al. 1996). Blair suggests an answer, using the constructs of working memory capacity and executive function (see also Kane \& Engle 2002). Indeed, the notions that working memory capacity and executive function are explanations of fluid intelligence are plausible. After all, the solution of fluid intelligence tasks undoubtedly involves the use of working memory. Similarly, executive functions are the result of an evolutionary recent brain area, so equating the operation of this brain area with fluid intelligence, again a capacity that is most evident in humans, would again seem plausible. It is also logical to identify fluid function with the prefrontal cortex, an area that is notable for playing a control function and not having direct connections with sensory input.

However, though the answer Blair gives has been suggested in the past, it is endorsed by relatively few current researchers. One reason for the lack of support for a relationship between fluid intelligence $(\mathrm{gF})$ and working memory and executive function is that tasks that assess working memory and executive function often do not reflect $\mathrm{gF}$. For instance, tasks developed according to working memory principles often do not correlate with $\mathrm{gF}$. Researchers arguing for a working memory capacity explanation of intelligence have then sought to strengthen this relationship by simply making working memory tasks involve the manipulation and transformation of information, elements that are commonly involved in fluid intelligence tasks (see Kyllonen \& Christal 1990). However, this would then suggest that it is not working memory capacity per se that is leading to the correlations between these tasks and fluid intelligence, and leads to a circular argument. Unsworth and Engle (2005) also found that a working memory capacity task predicted performance equally on Raven's problems that varied based on difficulty, memory load, and rule type. This again suggests that it is not working memory capacity per se that mediates the relationship between working memory capacity and fluid intelligence. Similarly, performance on executive function tasks often are not related to performance on fluid 